\title{
THEMIS and ground-based observations of successive substorm onsets following a super-long growth phase
}

\author{
J.-M. Liu ${ }^{1,2}$, Y. Kamide ${ }^{1}$, B.-C. Zhang ${ }^{1}$, H.-Q. Hu ${ }^{1}$, and H.-G. Yang ${ }^{1}$ \\ ${ }^{1}$ SOA Key Laboratory for Polar Science, Polar Research Institute of China, Shanghai, 200136, China \\ ${ }^{2}$ Sate Key Laboratory of Space Weather, Chinese Academy of Sciences, Beijing 100190, China \\ Correspondence to: J.-M. Liu (liujunming@pric.gov.cn) \\ Received: 14 June 2012 - Revised: 10 March 2013 - Accepted: 16 April 2013 - Published: 6 May 2013
}

\begin{abstract}
We present four successive substorm events, which followed a super-long, as long as $9 \mathrm{~h}$, growth phase on 5 December 2008, observed by the Time History of Events and Macroscale Interaction during Substorms (THEMIS) and the GOES 11 satellite with simultaneous coverage by the Alaska and THEMIS ground magnetometers. Several interesting and unique features were found for these cases. The interplanetary magnetic field was steadily southward and the solar wind speed was slow, less than $450 \mathrm{~km} \mathrm{~s}^{-1}$, which are thought to drive the long growth phase for the following onsets. At least four substorm expansion onsets occurred, including a double-onset event, which appears to be a challenge to the reconnection hypothesis for double-onset substorm and favored an instability mechanism for the onsets and could not be explained by the two neutral line models. For the onsets at 09:32 UT and 09:42 UT, the dipolarization signature was observed by GOES 11, which was located earthward of THEMIS C and THEMIS B. THEMIS $\mathrm{C}$ satellite caught a delayed and much weaker signature 13 min after GOES 11. THEMIS B observed no relating signature. These observations provide us with direct evidence that these events initiated at the near-earth region. The observations of THEMIS C and THEMIS B around the onsets favor the near-earth instabilities model for substorm onset.
\end{abstract}

Keywords. Magnetospheric physics (storms and substorms)

\section{Introduction}

Substorm is a characteristic disturbance in the magnetosphere-ionosphere system. A typical, isolated substorm goes through three phases: growth phase, expan- sion phase, and recovery phase (e.g., McPherron, 1979). During the growth phase lasting typically about one hour, enhanced magnetotail stretching occurs due to the dayside reconnection caused by the southward interplanetary magnetic field (IMF). This has become a consensus for isolated substorms in the substorm community. Meanwhile, the cross-tail current intensifies in the near-earth equatorial plasma sheet, and the magnetic field in the plasma sheet becomes increasingly stretched, forming a more tail-like configuration. The recovery phase is the time when the magnetosphere recovers to a quiet state again.

The substorm expansion onset is manifested as several signatures in the near-earth space, including the injection of energetic particles, the formation of a current wedge, auroral breakups, high-latitude magnetic perturbations, and fast flows in the magnetotail. The relative time sequences of these are usually used to identify how and where a substorm is initiated. Over the past four decades, two prevailing and competing scenarios, near-earth neutral line (NENL) model (e.g., Baker et al., 1996) and the current disruption (CD) model (e.g., Lui, 1996), have been proposed to account for the initiation and the subsequent sequence of the disturbances in the magnetotail associated with substorms. The NENL model requires the neutral line formation at a distance of $\sim 20-30 R_{\mathrm{E}}$ as the cause of the substorm expansion onset. All subsequent disturbances both closer to and farther from the earth follow after the reconnection. In the CD model, on the other hand, substorm expansion onset must begin with the instabilities and cross-tail current sheet collapse at $\sim 8-10 R_{\mathrm{E}}$, followed by the magnetic reconnection in the magnetotail. Both of the two models have found their supporting evidence by case studies (e.g., Angelopoulos et al., 2008; Lui, 2008), 


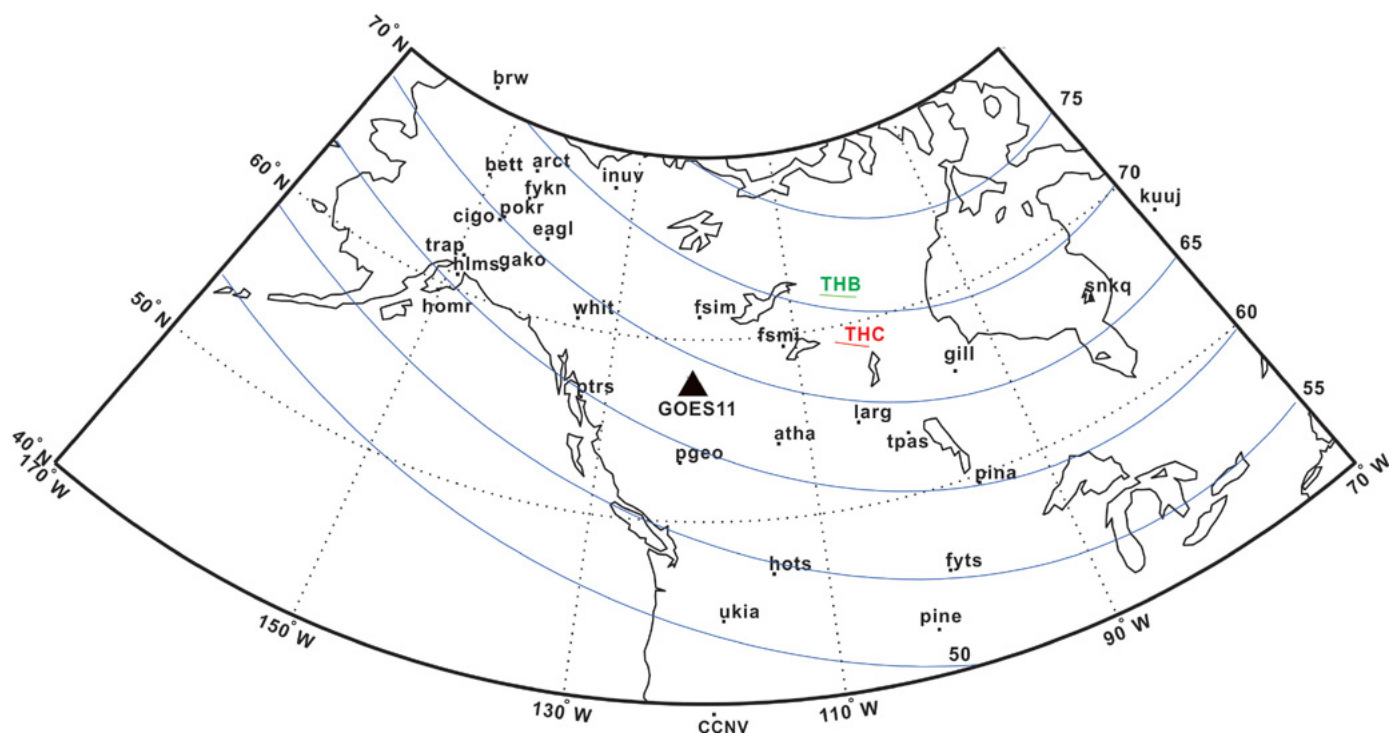

Fig. 1a. Foot point location of THEMIS B, THEMIS C, and GOES 11, and selected ground-based magnetic observatories for THEMIS mission and Alaska-chain observatories. The solid blue line represents the AAGCM latitude.
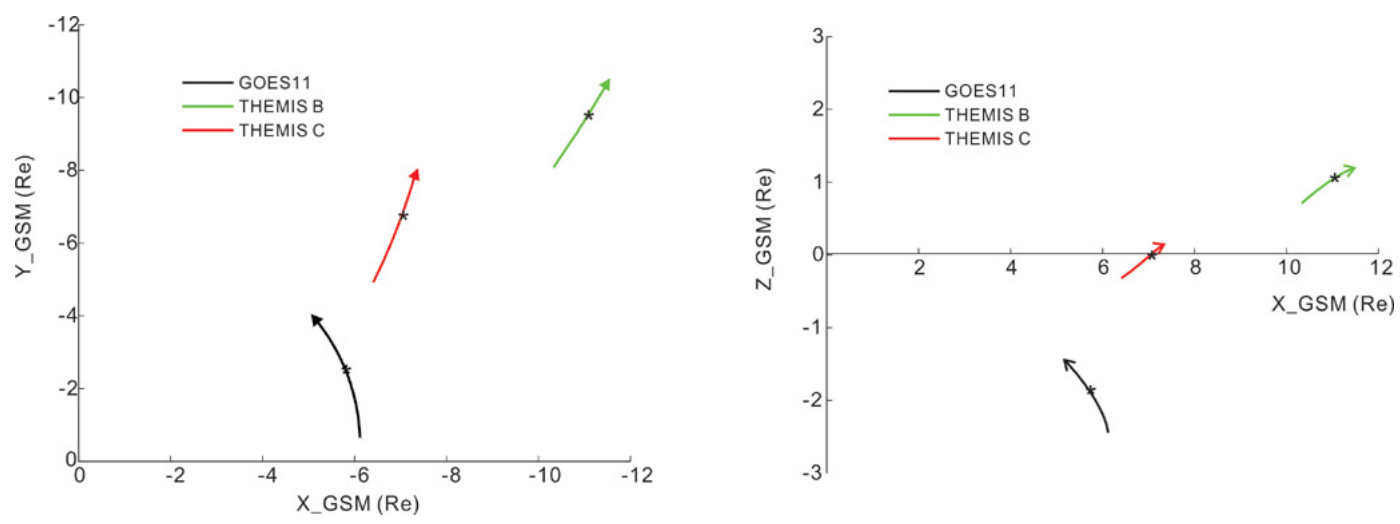

Fig. 1b. GOES 11, THEMIS B, and THEMIS C orbits in GSM coordinate between 09:00 UT and 11:00 UT on 5 December 2008. The arrows show the moving direction of the satellites. * represents the location of the satellites at 10:00 UT.

depending on the precise definition and the timing accuracy of the substorm signatures in various types of observations.

Dipolarization, a topological change of the plasma sheet from a stretched (tail-like) configuration to a relaxed (dipolelike) magnetic field configuration, has been well known as a typical signature of magnetospheric substorms in the 6$10 R_{\mathrm{E}}$ range (Lui, 1991). According to the substorm current wedge model, dipolarization is associated with the disruption and diversion of part of the magnetospheric cross-tail current into the auroral ionosphere (Atkinson, 1967; McPherron et al., 1973), where it flows across the substorm central meridian in the westward electrojet. Dipolarization begins in a longitudinally confined region and is followed immediately by a tailward and azimuthal expansion in both directions after onset (Kokubun and McPherron, 1981; Nagai, 1982), which is accompanied by a poleward and azimuthal expansion of the westward auroral electrojet in the midnight sector (Lopez et al., 1990).

In this paper, we present several unique substorm onsets that successively occurred following a super-long growth phase on 5 December 2008. Dipolarization signatures and high-latitude magnetic perturbations during these events have been investigated. Figure 1a shows the footprints of satellites used in this study, including GOES 11, THEMIS B, and THEMIS C, plotted along with THEMIS ground observatories and the Alaska chain stations, while Fig. $1 \mathrm{~b}$ presents the orbit position of the satellites. The magnetic field on the ground showed several noticeable enhancements of the westward electrojet during the entire interval that we are interested in. Meanwhile, the THEMIS B and THEMIS C probes were orbiting in the post-midnight sector. The geostationary satellite GOES 11 was also traversing in the post-midnight 

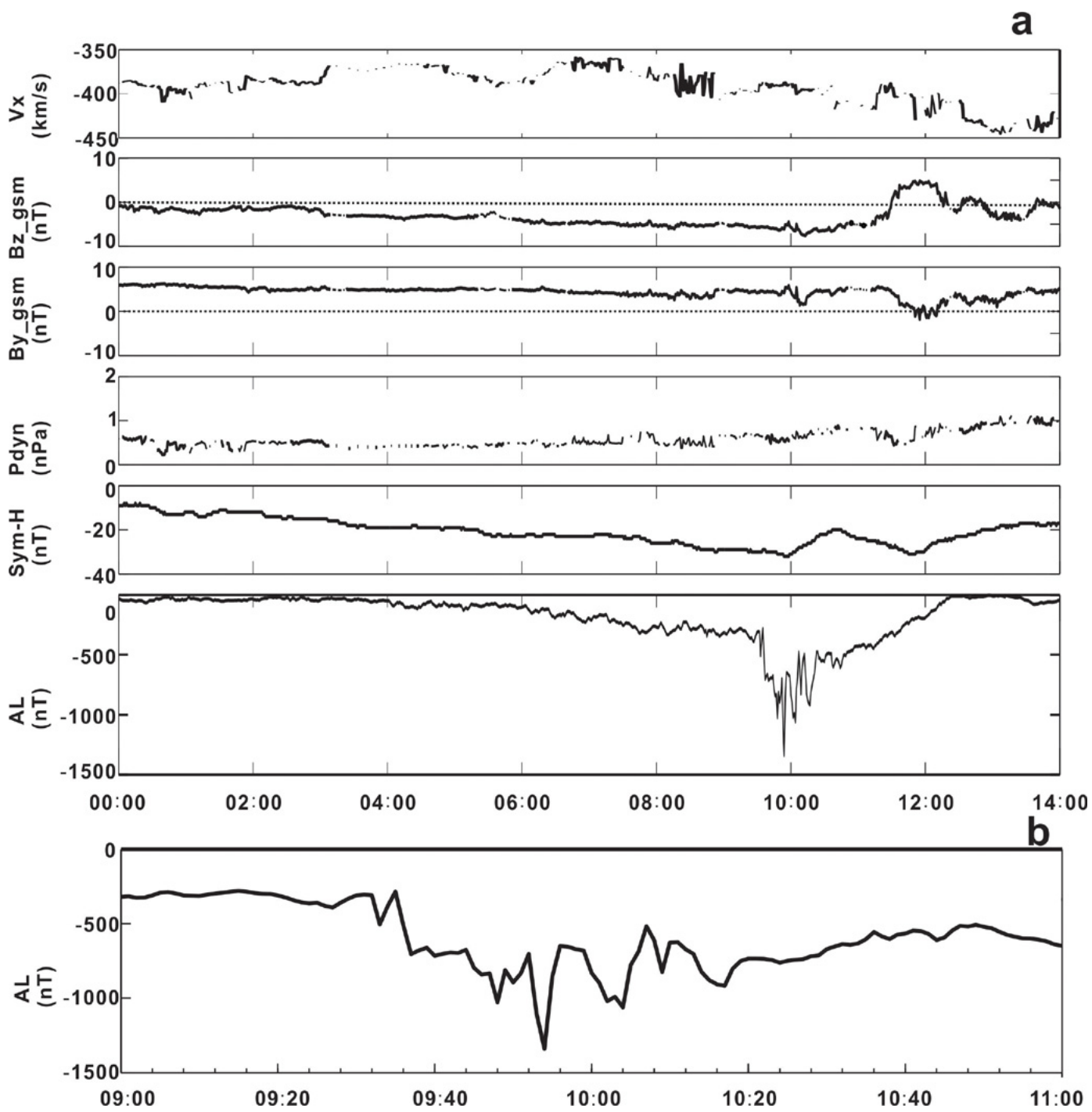

Fig. 2. (a) Stack plots of the solar wind parameters (including the solar wind speed, the $B_{\mathrm{Z}}$ and $B_{\mathrm{y}}$ components of the interplanetary magnetic field in GSM coordinates, and the dynamic pressure), Sym-H index, and the AL index from 00:00 UT to 14:00 UT on 5 December 2008. (b) The AL index from 09:00 UT to 11:00 UT.

sector, westward of the THEMIS B and THEMIS C satellite. Multiple dipolarizations were observed by the probes. By comparing the relative time sequence of the substorm events and their associated signatures, we analyze the substorm initiation position and possible mechanism for the events.

\section{Growth phase observations}

From top to bottom in Fig. 2a, the IMF and solar wind parameters obtained from the WIND satellite, the Sym-H and AL index are shown for the period between 00:00 UT and 14:00 UT on 5 December 2008. WIND data, downloaded from the OMNI website (http://omniweb.gsfc.nasa. gov/ow_min.html), have been shifted taking into consideration the travel time of the solar wind from the spacecraft position to the nose of Earth's bow shock. Figure 2b shows the AL index between 09:00 UT and 11:00 UT, when several substorm onsets occurred. As shown in Fig. 2a, the IMF was southward and experienced a gradual decrease in the period 00:00-10:00 UT. The magnitude of the solar wind is below $450 \mathrm{~km} \mathrm{~s}^{-1}$. No abrupt changes were seen in the solar wind and IMF conditions during that period. Associated with the southward IMF, a super-long growth phase for the following substorms began in conjunction with a gradually decreasing AL index. The AU index kept a value of less than $100 \mathrm{nT}$ for most of the growth phase time. Sym-H was larger than $-40 \mathrm{nT}$. The growth phase lasted for more than $9 \mathrm{~h}$ until 09:32 UT, at which time one substorm onset occurred that was identified as sudden drops in AL, as seen in Fig. 2b. The super-long growth phase could be also seen from the gradual 


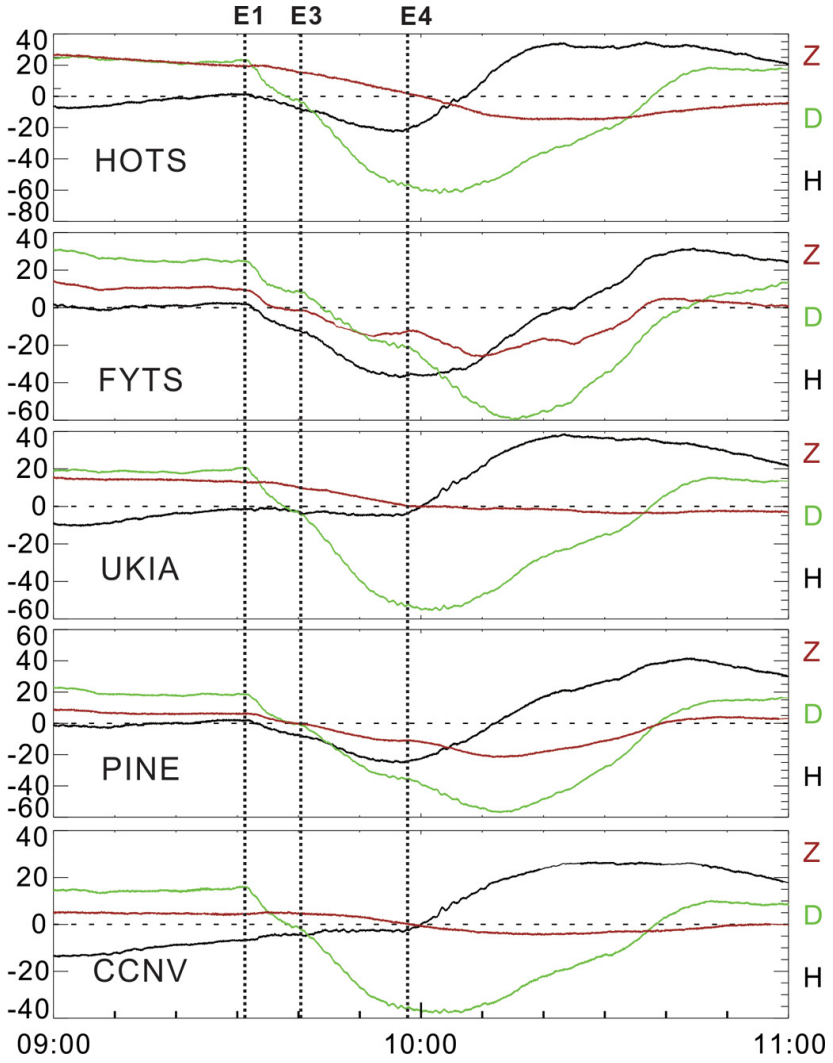

Fig. 3. Mid-latitude observations of the ground magnetic field: decrease of the D-component occurred three times, at about 09:32 UT, 09:41 UT, and 09:58 UT, respectively.

increase of the polar cap index, which indicates a gradual increase of the polar cap potential.

\section{Substorm expansion phase observations}

\subsection{Ground-based observations}

Figure 3 shows the three-component magnetograms that were observed at the mid-latitude magnetometer stations between 09:00 UT and 11:00 UT. As inferred from changes in the D-component, three enhancements of the downward field-aligned current could be clearly seen, two of which were observed by most of the stations at 09:32 UT and 09:42 UT. The third one, however, was observed only by station FYTS and PINE at 09:58 UT.

Figure 4 shows the time series of the $\mathrm{H}-\left(B_{\mathrm{H}}\right), \mathrm{D}-\left(B_{\mathrm{D}}\right)$, and $\mathrm{Z}-\left(B_{\mathrm{Z}}\right)$ components of the magnetic field observed by THEMIS ground-based and Alaska chains of magnetometers between 09:00 UT and 11:00 UT on 5 December 2008 . Corresponding to the three enhancements of the field-aligned current, three obvious sharp decreases of $B_{\mathrm{H}}$ were also recorded. As shown in Fig. 3a, the first one (E1) occurred at 09:32 UT, covered by most of the Alaska stations as seen from the sharp decrease of $B_{\mathrm{H}}$ associated with a strong westward electrojet. At the same time, stations eastward of the Alaska stations, such as WHIT, PGEO, ATHA, TPAS, and PINA, caught the similar signatures, as seen from Fig. 4b. According to the relative changes of $B_{\mathrm{Z}}$, the center of the westward electrojet lied northward of stations POKR, CIGO, and GAKO, and southward of stations EAGL and BETT. Just 3 min after E1, at about 09:35 UT, a significant decrease of $B_{\mathrm{H}}$ took place again and lasted more than $30 \mathrm{~min}$ at several Alaska stations, such as POKR, CIGO, EAGL, and TRAP. According to the $B_{\mathrm{Z}}$ changes, this substorm initiated southward of POKR and CIGO.

As shown in Fig. 4b, at 09:42 UT, when the second enhancement of the field-aligned current occurred, a further decrease of $B_{\mathrm{H}}$ (E3) occurred at stations PGEO, ATHA, TPAS, and PINA. At 09:58 UT, one more sudden decrease of $B_{\mathrm{H}}$ (E4) was observed at stations FSMI, GILL, KUUJ, and NAIN.

\subsection{Dipolarizations observed by the GOES 11 and THEMIS satellites}

The GOES 11 satellite was located nearly at $(6.0,1.6$, -2.2) $R_{\mathrm{E}}$ in geocentric solar magnetospheric system (GSM) coordinates at 09:32 UT. As shown in Fig. 5, it could been clearly seen that increases of the z-component of the magnetic field $\left(B_{\mathrm{Z}}\right)$ took place at 09:34 UT and 09:43 UT, accompanied by turbulence of the y-component, which indicated quick changes of the field-aligned currents. According to the time sequence, we suggest that these three dipolarizations were associated with E1 and E3. For E2, GOES 11 satellite observed no obvious dipolarization, which we think was due to the short time difference between E1 and E2. The dipolarization for E1 continued to develop when E2 occurred, which make the signature of E2 inconspicuous. For E4, GOES 11 observed no obvious dipolarization signature, which was due to the fact that GOES 11 was located outside the current wedge associated with $\mathrm{E} 4$, as seen from the middle-latitude observations in Fig. 3. Only stations FYTS and PINE observed the magnetic bays associated with E4, while stations HOTS, UKIA, and CCNV, which were located westward of stations FYTS and PINE, observed no related signatures. GOES 11 was located even westward of stations HOTS, UKIA, and CCNV and should not observe the dipolarization associated with $\mathrm{E} 4$.

THEMIS C was located at $\sim(-6.9,-6.4,-0.06) R_{\mathrm{E}}$ in GSM coordinates at 10:00 UT. Figure 6 shows THEMIS C observations of the magnetic field $\left(B_{\mathrm{x}}, B_{\mathrm{y}}\right.$, and $B_{\mathrm{Z}}$ components in GSM coordinate, as well as the total field), plasma $\beta$, electron flux, ion and electron temperature from 09:00 UT to 11:00 UT on 5 December 2008. Before 10:00 UT, the ion and electron number density was about $0.4 \mathrm{~cm}^{-3}$, ion temperature about $4 \mathrm{keV}$, electron temperature about $1 \mathrm{keV}$, the plasma $\beta$ about 0.1 , and the magnetic field elevation angle about 20 degrees. These parameters indicate that 

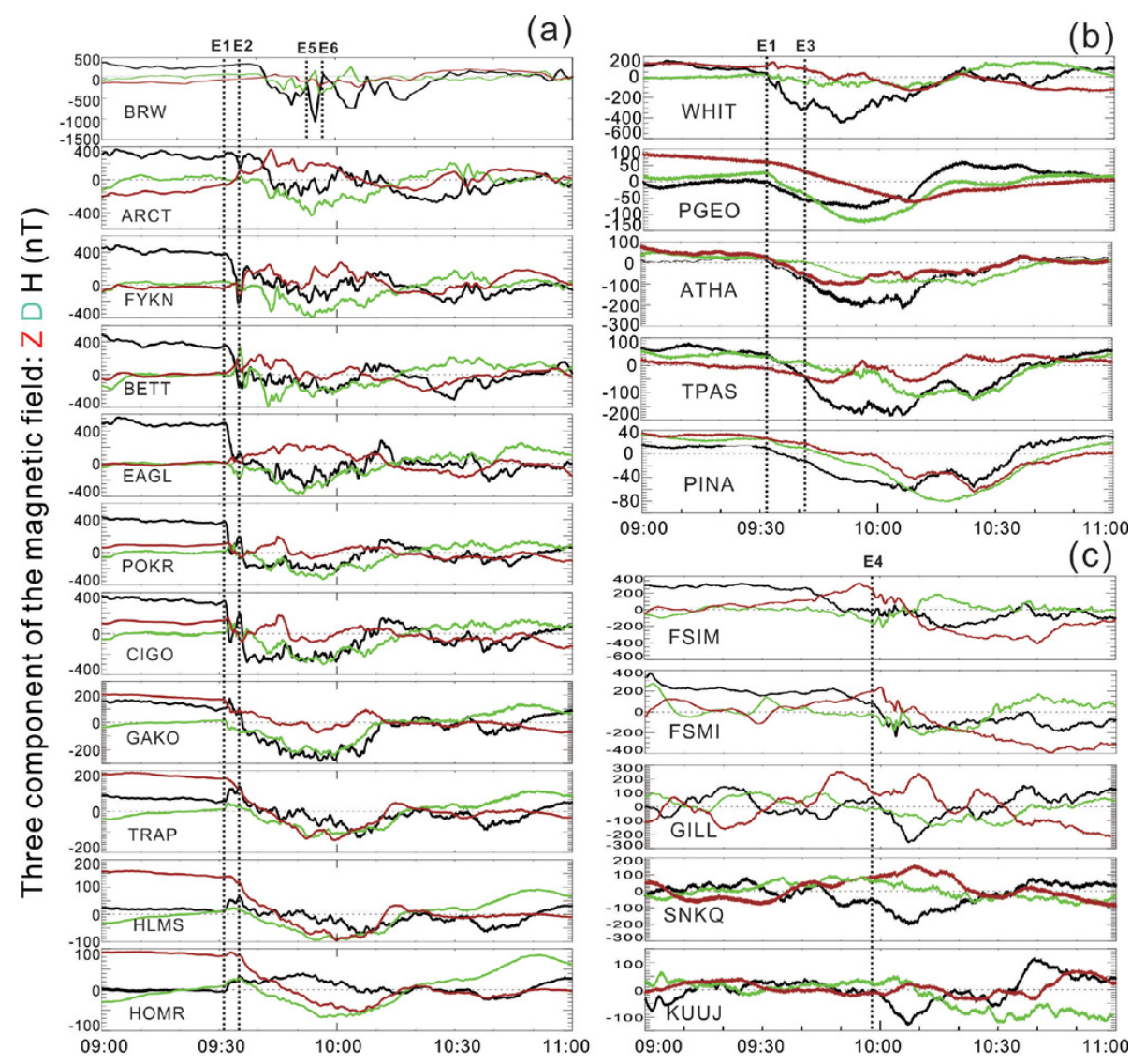

Fig. 4. The H-, D-, and Z- component magnetograms from selected THEMIS and Alaska magnetometers from 09:00 to 11:00 UT on 5 December 2008.

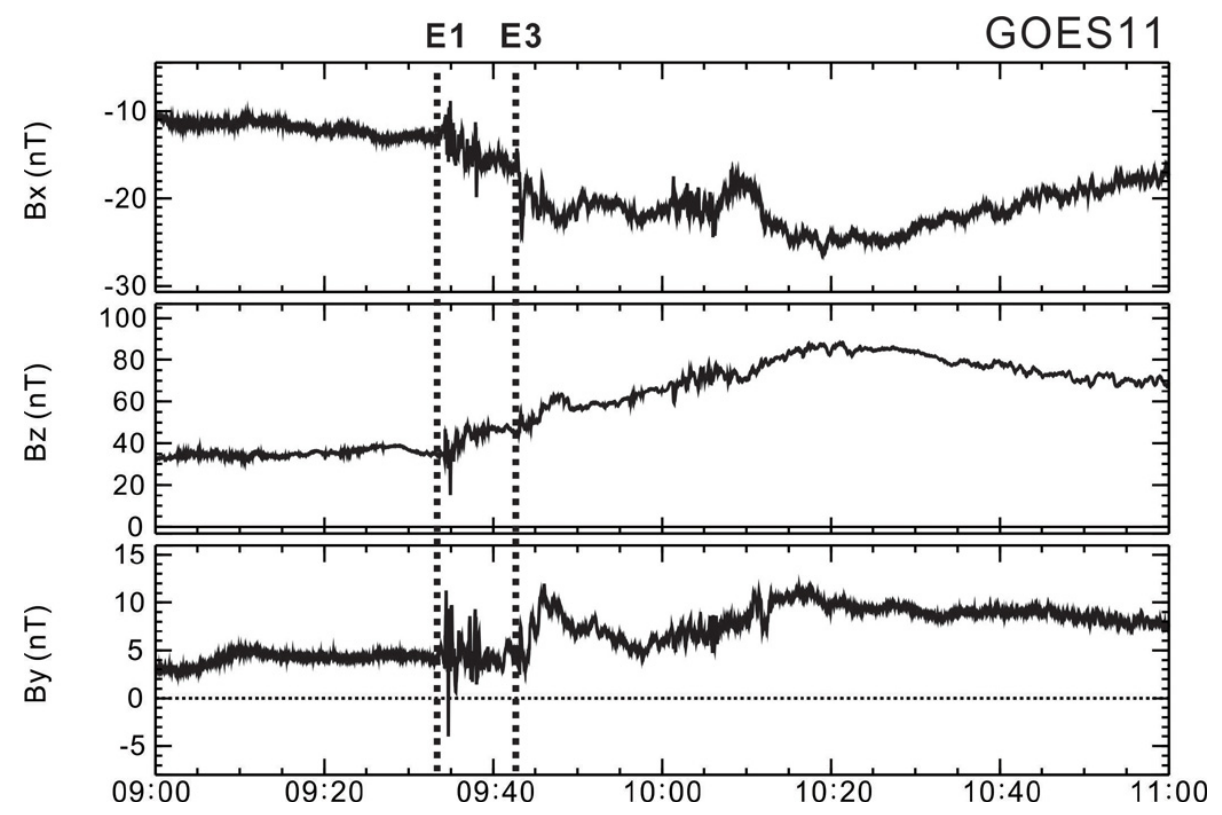

Fig. 5. Three components of the magnetic field in GSM coordinate observed by GOES 11 satellite between 09:00 UT and 11:00 UT on 5 December 2008. 


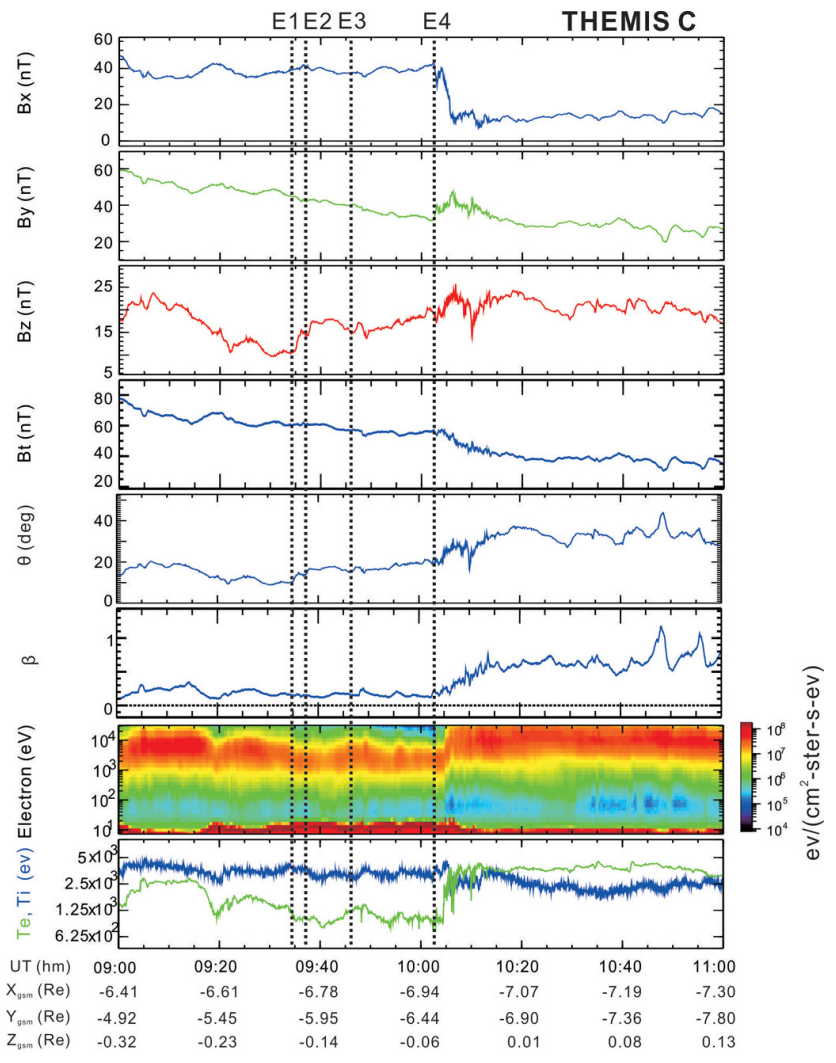

Fig. 6. Summary plots of THEMIS C's observations between 09:00 UT and 11:00 UT on 5 December 2008: the three components of the magnetic field in GSM coordinate, the total magnetic field, plasma $\beta$, energy spectra of $0.005-300 \mathrm{keV}$ electrons from the Solid State Telescope (SST) and ESA instruments, and the ion and electron temperature.

THEMIS C was located in an outer portion of the central plasma sheet. Four obvious increases of $B_{\mathrm{Z}}$ could be seen at about 09:35 UT, 09:37 UT, 09:46 UT, and 10:03 UT. The first three were accompanied by a gradual decrease of $B_{\mathrm{y}}$ and a relatively steady $B_{\mathrm{x}}$, and the magnetic field elevation angle $\theta$ showed a slight increase. For the third one at 10:03 UT, the magnetic field elevation angle $\theta$ started another obvious increase, which indicates the magnetic dipolarization at THEMIS C. At the same time, a sharp decrease of $\left|B_{\mathrm{x}}\right|$ and $B_{\mathrm{t}}$ appeared, representing a decrease of the cross-tail current. Meanwhile, the plasma density, the plasma temperature, and $\beta$ all suddenly jumped up, while the magnetic pressure (not shown) was reduced, implying a quick expansion of the plasma sheet. According to the time sequence of the dipolarizations and the enhanced westward electrojet, we contend that these four dipolarizations correspond to E1, E2, E3, and $\mathrm{E} 4$, respectively.

THEMIS B was located nearly at $(-10.93,-9.21$, $-1.0) R_{\mathrm{E}}$ in GSM coordinates at 10:00 UT, tailward of THEMIS C. Figure 7 shows THEMIS B observations of the magnetic field, plasma $\beta$, electron flux, ion and electron tem-

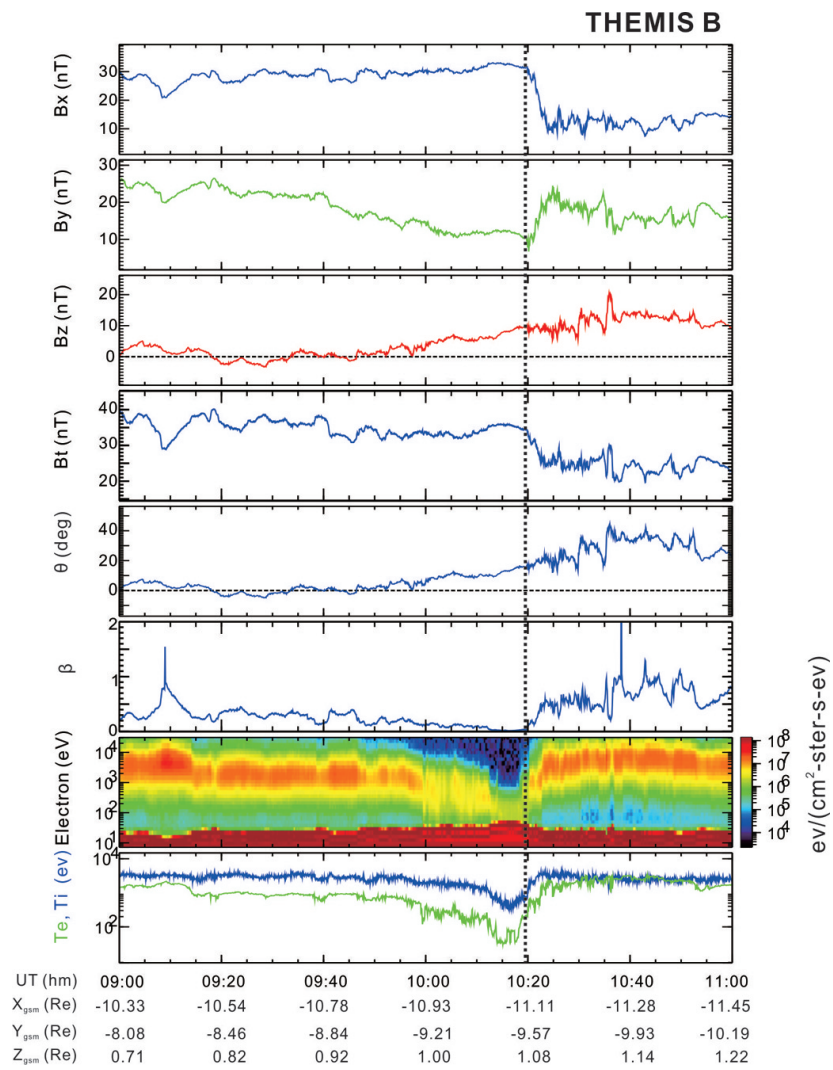

Fig. 7. Summary plots of THEMIS B's observations between 09:00 UT and 11:00 UT on 5 December 2008: the three components of the magnetic field in GSM coordinate, the total magnetic field, plasma $\beta$, energy spectra of $0.005-300 \mathrm{keV}$ electrons from the SST and ESA instruments, and the ion and electron temperature.

perature from 09:00 UT to 11:00 UT on 5 December 2008 . The electron number density was about $0.2 \mathrm{~cm}^{-3}$, ion temperature about $1 \mathrm{keV}$, electron temperature about $0.3 \mathrm{keV}$, the plasma $\beta$ about 0.1 , and the magnetic field elevation angle about 10 degrees. These parameters indicate that THEMIS B was located in the outer part of the central plasma sheet. At about 10:20 UT, THEMIS B observed a similar signature as what THEMIS C observed at 10:03 UT, including a decrease of $B_{\mathrm{x}}$ absolute value, a clear increase of $B_{\mathrm{y}}$, an increased magnetic elevation angle and enhancement of the electron temperature. However, the ion temperature also shows a large enhancement, which is different from THEMIS C. This could be interpreted as the combined contribution of the dipolarization and the relative position of the satellites. For THEMIS C, the ion and electron kept steady before the dipolarization at 10:02 UT, which indicated that THEMIS C was located at the plasma sheet during the whole period of time. The enhancement of the electron temperature was mainly caused by the plasma sheet expansion process. For THEMIS B, one can see that the ion and electron temperature started a gradual decrease at 09:50 UT, implying that THEMIS B was moving away from the plasma sheet. When the dipolarization 
occurred, THEMIS B returned back to the plasma sheet because of the expansion of the plasma sheet. By comparing the ion and electron temperature before 09:50 UT and that after the dipolarization, no obvious enhancement could be seen for the ion temperature, but a clear enhancement of the electron temperature could be achieved, which implies that the ion temperature showed no enhancement in the distant plasma sheet following the plasma sheet expansion. The enhancement of the ion temperature observed by THEMIS B was mainly attributed to the relative change of the position of THEMIS B in the magnetotail. Therefore, THEMIS C and THEMIS B observed the same signature associated with the dipolarization, if we take the movement of the satellite into account.

\section{Discussion}

An interesting feature of the successive substorm on 5 December 2008 is the existence of the super-long growth phase, which lasted for more than $9 \mathrm{~h}$. As shown in the magnetic field observations of the GOES 11 satellite, the $B_{\mathrm{z}}$ at the nightside geostationary altitude, which is affected seriously by the cross-tail current, was only $33 \mathrm{nT}$, the minimum compared to the values during the ten days before and after $5 \mathrm{De}-$ cember 2008 (from 26 November to 16 December, not shown here). Moreover, the lobe magnetic field is unusually large. To see this in more quantitative terms, we have checked the empirical model of the lobe field at substorm onset, which Shukhtina et al. (2004) obtained on the basis of Geotail observations. For the THEMIS B position and given solar wind conditions, the model predicts the lobe field value of $24 \mathrm{nT}$, while the actual value in our case is $33 \mathrm{nT}$. The extraordinary large lobe field and small $B_{\mathrm{Z}}$ at the geostationary orbit indicated the existence of very strong cross-tail currents, hence, an unusual loading process before the following substorm expansions, which probably results in several successive substorms, as shown above.

Then, one immediate question arises as to why no substorm occurred during the long southward IMF period before the onsets we identified. Previous studies (e.g., Rostoker et al., 1983) have shown that the disturbances in the solar wind/IMF could trigger substorm. There exists a relatively stronger loading process for the substorm that occurred during a steady solar wind/IMF condition (Liu et al., 2011). Hsu and McPherron (2004) suggested the internal instability in the magnetosphere that causes the substorm is susceptible to external perturbations in the solar wind/IMF. For the time period in which we are interested in this work, the solar wind and IMF were rather steady and showed no appreciable sudden changes during the growth phase. The solar wind velocity was also slow (below $450 \mathrm{~km} \mathrm{~s}^{-1}$ ). We thus propose that the quiet solar wind and IMF conditions favored a relatively steady magnetosphere, where it is not easy for internal instability to develop to cause a substorm.
We believe that our work also presents another piece of evidence for the coexistence of two substorms in the same longitudinal sector, as Kamide et al. (1977) identified. Both E1 and E2 occurred in the Alaska longitude. The onset times of the two have only $3 \mathrm{~min}$ difference. In some previous studies (e.g., Cheng et al., 2005, 2011), this kind of events was named as a double-onset substorm, which was explained as the effect of the reconnection at the near-earth neutral point on closed field line within the plasma sheet and on the open flux of tail lobes. However, if that is really the case, the second onset should be at latitudes higher than the first one, which is not the case in our event. Moreover, for stations TRAP and HLMS, the currents in the polar ionosphere showed the eastward and westward directions for the two substorms, respectively, as seen from the $B_{\mathrm{H}}$ disturbance, which implies a southward current system for E2 in comparison with E1. Thus, our observations present a serious challenge to the reconnection hypothesis for double-onset substorms. We interpret our events according to the mechanism in which the instabilities responsible for substorm onset could occur anywhere in the plasma sheet. The most active region may expand tailward as a substorm progresses due to the expansion of the plasma sheet. Therefore, if there is one more substorm followed, the second one is most likely to occur at a higher latitude. However, this interpretation cannot exclude totally the possibility that the second onset occurs at a lower latitude, which is exactly the case in this study. Instabilities here mean some actions that tend to trigger expansion onset, such as the current disruption in the plasma sheet. However, which kind of instabilities trigger the onsets is beyond the scope of this work.

For events E1 and E3, the dipolarization signatures were observed by GOES 11 and THEMIS C at 09:34/09:43 UT and 09:35/09:46UT, respectively. The signature observed by THEMIS $C$ was much weaker than that by GOES 11 . Moreover, THEMIS B, which was located tailward of GOES 11 and THEMIS C, observed no associated signature. These observations clearly indicate that E1, E2, and E3 initiated earthward of THEMIS B, which were located at the near-earth plasma sheet. We suggest that the near-earth initiation of the substorm onset is the one that appears to be realistic. For E4, the associated dipolarization signature was first observed by THEMIS C, as compared to that observed by THEMIS B, suggesting that E4 initiated earthward of THEMIS B (i.e., the near-earth region). Thus, most of the onsets shown above favor the near-earth initiation. A few supporting results for near-earth initiation of substorms have been published earlier based on data for individual substorm onset (e.g., Lui, 2008; Mende et al., 2009), which depend on a critical mapping of the configuration of the magnetic field in the magnetotail and its footprints in the ionosphere, or an accurate determination of the onset time of substorm expansion onset. However, this is not the case for this study. An accurate mapping from the magnetosphere to the ionosphere is not so required, and a 
rough estimate of the onset time is enough for the present purpose.

One last point that should be emphasized is that THEMIS B observed no special signature before E4, although THEMIS C and THEMIS B were located at a similar magnetic local time (MLT) position as the onset position. Many studies have shown that reconnection takes place in the mid-tail before a substorm expansion onset is observed on the ground. However, this is not the case for the events studied in this paper. THEMIS C and THEMIS B observed no reconnection-related signature before the events occurred. Therefore, our observations again present a serious challenge to the standard hypothesis that magnetic reconnection in stretched magnetotail fields triggers onset. The events in this paper favor the near-earth instabilities model for substorm onset, which does not presuppose NENL formation as necessary for a typical substorm, as proposed by Ohtani (2001).

There exist another two clear large drops in $\mathrm{AL}$ at about 09:52 UT and 09:56 UT respectively. One may treat these as two large substorms. However, most of the ground stations did not observe the signature, except the station BRW (E5 and E6 in Fig. 3a). In addition, neither dipolarization nor field-aligned current enhancement was observed, which implies these two AL decreases reflect a localized disturbance, but not substorm expansions.

\section{Summary}

Four successive substorm events, which followed a superlong, as long as $9 \mathrm{~h}$, growth phase on 5 December 2008, were observed by the THEMIS and the GOES 11 satellites with simultaneous coverage by the Alaska and THEMIS ground magnetometers. Several interesting and unique features were found for these cases. The interplanetary magnetic field was steadily southward and the solar wind speed was slow, which are thought to account for the absence of substorm expansion onsets during the long southward IMF period. A doubleonset event favored an instability mechanism for the onsets and could not be explained by the two neutral line models. By comparing the relative responses of the substorms on the ground and in the magnetosphere, we found that these substorm events initiated in near-earth magnetotail. Internal instabilities in the magnetosphere seem to be responsible for the occurrence of the substorm expansions.

Acknowledgements. This work at PRIC was supported by the National Basic Research Program of China (No. 2012CB825603) and by the National Natural Science Foundation of China under grants 41104090, 41031064, 40874082, and 40890164, the Ocean Public Welfare Scientific Research Project, the State Oceanic Administration People's Republic of China (No. 201005017), the Polar Strategic Research Foundation of China (No. 20100202), and Project Supported by the Specialized Research Fund for State Key
Laboratories. We acknowledge NASA contract NAS5-02099 and V. Angelopoulos for the use of data from the THEMIS Mission. We also thank C. W. Carlson and J. P. McFadden for use of ESA data, K. H. Glassmeier, U. Auster and W. Baumjohann for the use of FGM data. The AL and Sym-H indices were provided by the World Data Center C2 for Geomagnetism, Kyoto University. We also thank the OMNI web for the IMF data at the Earth nose (see http//omniweb.gsfc.nasa.gov/ow_min.html).

Topical Editor I. A. Daglis thanks two anonymous referees for their help in evaluating this paper.

\section{References}

Angelopoulos, V., McFadden, J. P., Larson, D., Carlson, C. W., Mende, S. B., Frey, H., and Phan, T.: Tail reconnection triggering substorm onset, Science, 321, 931-935, doi:10.1126/science.1160495, 2008.

Atkinson, G.: An approximate flow equation for geomagnetic flux tubes and its application to polar substorms, J. Geophys. Res., 88, 5373-5382, 1967.

Baker, D. N., Pulkkinen, T. I., Angelopoulos, V., Baumjohann, W., and McPherron, R. L.: Neutral line model of substorms: Past results and present view, J. Geophys. Res., 101, 12975-13010, 1996.

Cheng, C. C., Russell, C. T., Reeves, G. D., Connors, M., and Moldwin, M. B.: On the relationship between double-onset substorm, pseudobreakup, and IMF variation: The September 1999 event, J. Geophys. Res., 110, A07201, doi:10.1029/2004JA010778, 2005.

Cheng, C.-C., Russell, C. T., Angelopoulos, V., Mann, I. R., Glassmeier, K.-H., and Baumjohann, W.: THEMIS observations of double-onset substorms and their association with IMF variations, Ann. Geophys., 29, 591-611, doi:10.5194/angeo-29-5912011, 2011.

Hsu, T. S. and McPherron, R. L.: Average characteristics of triggered and nontriggered substorms, J. Geophys. Res., 109, A07208, doi:10.1029/2003JA009933, 2004.

Kamide, Y., Akasofu, S. I., and Rieger, E. P.: Coexistence of two substorms in the midnight sector, J. Geophys. Res., 82, 1620 1624, 1977.

Kokubun, S. and McPherron, R. L.: Substorm signatures at synchronous altitude, J. Geophys. Res., 86, 11265-11277, 1981.

Liu, J.-M., Zhang, B.-C., Kamide, Y., Wu, Z.-S., Hu, Z.-J., and Yang, H.-G.: Spontaneous and trigger-associated substorms compared: Electrodynamic parameters in the polar ionosphere, J. Geophys. Res., 116, A01207, doi:10.1029/2010JA015773, 2011.

Lopez, R., Sibeck, D., McEntire, R., and Krimigis, S.: The energetic ion substorm injection boundary, J. Geophys. Res., 95, 109-117, 1990.

Lui, A. T. Y.: A synthesis of magnetospheric substorm models, J. Geophys. Res., 96, 1849-1856, 1991.

Lui, A. T. Y.: Current disruption in the Earth's magnetosphere: Observations and models, J. Geophys. Res., 101, 13067-13088, 1996.

Lui, A. T. Y.: Determination of the substorm initiation region from a major conjunction interval of THEMIS satellites, J. Geophys. Res., 113, A00C04, doi:10.1029/2008JA013424, 2008.

McPherron, R. L.: Magnetospheric substorms, Rev. Geophys. Space Phys., 17, 657-681, doi:10.1029/RG017i004p00657, 1979. 
McPherron, R. L., Russell, C. T., and Aubry, M.: Satellite studies of magnetospheric substorms on August 15, 1968, 9. Phenomenological model for substorms, J. Geophys. Res., 78, 3131-3149, 1973.

Mende, S., Angelopoulos, V., Frey, H. U., Donovan, E., Jackel, B., Glassmeier, K.-H., McFadden, J. P., Larson, D., and Carlson, C. W.: Timing and location of substorm onsets from THEMIS satellite and ground based observations, Ann. Geophys., 27, 28132830, doi:10.5194/angeo-27-2813-2009, 2009.

Nagai, T.: Observed magnetic substorm signatures at synchronous altitude, J. Geophys. Res., 87, 4405-4417, 1982.
Ohtani, S.-I.: Substorm trigger processes in the magnetotail: Recent observations and outstanding issues, Space Sci. Rev., 95, 347359, 2001.

Rostoker, G.: Triggering of expansive phase intensifications of magnetospheric substorms by northward turnings of the interplanetary magnetic field, J. Geophys. Res., 88, 6981-6993, 1983.

Shukhtina, M. A., Dmitrieva, N. P., and Sergeev, V. A.: Quantitative magnetotail characteristics of different magnetospheric states, Ann. Geophys., 22, 1019-1032, doi:10.5194/angeo-221019-2004, 2004. 